\title{
The Software Defined Transport Network: Fundamentals, Findings and Futures
}

\author{
Daniel King ${ }^{1}$, Charalampos Rotsos ${ }^{1}$, Alejandro Aguado ${ }^{2}$, Nektarios Georgalas ${ }^{3}$, and Victor Lopez ${ }^{4}$ \\ ${ }^{1}$ University of Lancaster, United Kingdom \\ ${ }^{2}$ University of Bristol, United Kingdom \\ ${ }^{3}$ British Telecom, United Kingdom \\ ${ }^{4}$ Telefónica Research and Development $(I+D)$, Spain
}

\begin{abstract}
The Software Defined Network (SDN) is an established network paradigm, architecture and principles, that attracted significant research effort in recent years. An SDN-enabled infrastructure decouples network control from forwarding and enables direct programming. Recently, there is an increasing effort to introduce SDN support in the transport layers of the network operators WAN infrastructure, like Layer 0 (WDM \& DWDM) and Layer 1 (SONET/SDH \& OTN) technologies. We refer to this infrastructure as the "Software Defined Transport Network", and benefits include network management devolvement, timely connectivity provision, improved scalability, and open and flexible programmability using well-defined API.

This paper outlines the main elements of Software Defined Transport Networks and highlights relevant Application-Based Network Operations (ABNO) enabling technologies. We demonstrate how this technology will benefit network operators, and provide an overview of research results and deployment examples. Finally, we identify some of the technology gaps and future research opportunities.
\end{abstract}

Keywords: software defined networks, application-based network operations, optical switching.

\section{INTRODUCTION}

High capacity Cloud application connectivity between network operator data-centres predominantly uses longlife, high-capacity, connectionless network services. Increasingly, there is a need for great network dynamicity and on-demand connectivity (scheduled) with guarantees, like bandwidth, bounded latency, controlled jitter, availability, and explicit control of network paths.

The proliferation of high bandwidth Internet and Cloud services, including high bandwidth multimedia content, office automation platforms, and distribution of gaming content, requires reliable connectivity between Data Centre (DC) sites. These WAN connections are high-capacity links. Any WAN failure typically affects all Internet and Cloud applications traversing the link, until the IP control plane can recover from the fault. Network operators, including British Telecom and Telefonica, have a widely diverse set of users with varying network traffic profiles and often requiring differentiated traffic treatment with varying levels of Quality of Service (QoS) and Quality of Experience (QoE) objectives. Both operators have many physical data centres across the globe. Each data centre connects the WAN backbone network via several 10GE/40GE/100GE links. Each DC accommodates 1000s of customers, who are the potential users of Data Center interconnection services.

This paper discusses the design, implementation, and evaluation of Software Defined Transport Network platforms underpinned by the Internet Engineering Task Force (IETF) Application-Based Network Operations (ABNO) framework [1]. ABNO represents a systematic approach to interconnect Internet and Cloud services, at scale, while ensuring key technical objectives, like deployment dynamicity, end-to-end connection assurance, centralised control and traffic engineering, automation.

\section{BACKGROUND}

Large network operators, like British Telecom and Telefonica, have to integrate multiple packet technologies allowing network infrastructure to deliver a variety of services to support the different characteristics and dynamic demands of high bandwidth Cloud applications. Increasingly, there is demand to make the network more efficient and responsive to service requests, creating connections on demand and for the specific time periods required. This differs from the established model where services in the network are instantiated in response to management commands driven by a human user using a wide variety of Operational Support Systems (OSS), and where networks are typically over-provisioned to ensure minimal traffic loss, even at peak traffic periods.

Traditionally, an operator may use dedicated physical links or complex distributed control plane mechanisms such as Generalized Multi-Protocol Label Switching (GMPLS) to meet the customer and service requirements. With the advent of SDN and the capability to provide centralise control of resources and using programmatic flow-based technologies, like OpenFlow, Cloud DC interconnection can fulfil the objectives described previously. Several technology approaches exist for introducing SDN and related technologies, including 
centralised control over distributed routing [2], and utilising these technologies into the transport network to accomplish the aforementioned objectives.

Software defined transport networking describes the network controller, managing the WAN links and the physical transmission/switching nodes, and the optical forwarding technology. Control and forwarding elements are separated and are connected by a common open interface (Application Programmable Interface); most network control and node/link intelligence, including resource description, modeling and abstraction, resides in centralised controller; and the physical hardware offers high level of flexibility to support different network demands and conditions.

The software defined transport network has some significant differences compared to packet-layer Software Defined Networking (SDN), mainly due to many practical analog physics attributes, including: wavelength continuity, non-linearity effects, signal-to-noise ratio and dispersion compensation. This makes it difficult to have a ubiquitous digital network layer networks. Therefore, multiple technology abstractions are required, and physical control-layer functions will need to be considered by the network controllers.

\subsection{Applied Research}

A survey conducted by Forrester Consulting on behalf of Juniper Network, January 2014 [3] identified network bandwidth, performance, reliability, automation/programmability as being key demands from Cloud infrastructure consumers. Given the existing and growing Cloud customer base, the deployment and modification of connections between Cloud consumers and operator datacenters is critical. Therefore, British Telecom and Telefonica identified the need to develop much more efficient optical-based transport network capability, without increasing control and management complexity. This included minimising the deployment time of new technologies in the network and reusing existing protocol knowledge where possible. Fulfilment of these objectives included the establishment and support of strategic European Commission funded FP7 research projects by both companies, including: IDEALIST, STRAUSS, DISCUS and LIGHTNESS.

\section{SOFTWARE DEFINED NETWORK TRANSPORT}

The adoption of SDN transport by British Telecom and Telefonica, and specifically a logically centralised controller principle, provided the cornerstone for the objectives for improved network usage efficiency and the foundation for further service innovation. We use the term logically centralised to signify that network control may appear focused in a single entity, independent of its possible implementation in distributed form. The centralised control principle states that resources can be used more efficiently when viewed from a global perspective. A network controller would have to combine a number of technology components, mechanisms and procedures, including:

- Access control of entities and applications requests for network resource information and connectivity,

- Gathering information about the resources available in a network,

- Controller and modification of variable bit-rate optical transponders and adaptive optical amplifiers,

- Consideration of traffic applications and how they would need to be mapped to underlying network resources,

- Handling of path computation requests and responses,

- Provisioning and reserving end-to-end network resources,

- Verification of end-to-end connections,

- Ongoing resource optimisation.

The software defined transport network controller would also need to provide resource orchestration capabilities that span a number of subordinate domains and/or in cooperation with other entities, and thereby offer resource efficiency when setting up end-to-end services and overall operation of network resources used to provide those end-to-end services.

\section{SOFTWARE DEFINED NETWORK TRANSPORT PLATFORM}

\subsection{Application-Based Network Operations (ABNO)}

The three tenants of SDN are programmability, control and data plane separation, and logically centralised management of ephemeral network state, regardless of the degree of centralisation. In an ideal world, it should be possible to utilise a distributed control plane as well, providing the best practices of centralised control, and distributed control plane for ephemeral state management.

An objective of the software defined transport network control platform was to reuse existing network standards when possible. The IETF's SDN Framework Application-Based Network Operations (ABNO) provides a reference architecture for our controller development. ABNO was designed using the following architectural principles: 
1. Loose Coupling: For ease of implementation and fast development, it does not attempt to tightly integrate the functional components of the network controller. Instead, it provides well-defined and standardsbased candidate APIs and protocol mechanisms.

2. Low Overhead: The framework ensures that each management and control function is not duplicated, reducing the overall controller platform overhead.

3. Modular: A modular design enables easier composition of existing features into new capabilities.

4. Intelligent: Designing the ABNO framework around the Path Computation Element (PCE) and Traffic Engineered (TE) principles, provides significant benefits for controlling a range of network technologies and maximising resource utilisation.

5. Resource Management: The ABNO framework allows state discovery and management for various network and node types. This state is collected using protocol mechanisms provided by traditional and existing network and service management tools.

6. Dynamic Management: A key goal for an SDN controller is dynamic control based on application demands and other network events.

7. Policy Control: It is important to implement policy management to provide the mechanisms for specifying connection requirements (e.g., TE-constraints, security) for various applications. It also allows operators to associate different service levels.

8. Technology Agnostic: The ABNO framework communicates with the network nodes using a variety of Southbound APIs and protocols. Allowing for a wide variety of forwarding mechanisms to be managed using ABNO.

Ideally, a software defined transport network controller would also need to address common functions like addressing and routing, topology discovery, network abstraction, path computation, and connection provisioning. In the context of our controller design, the use of a control plane fulfils the requirements of reusing technology and automatic end-to-end provisioning and rerouting of connections, while supporting different levels of quality of service. From a high level perspective, and similarly to any software system that automates tasks and processes, the functions of a control plane may be distributed or centralised.

\subsection{Implementations}

ABNO has been successfully demonstrated for a variety of transport network operations, including but not limited to:

- $\quad$ ABNO: A feasible SDN approach for multi-vendor IP and optical networks [4];

- ABNO-based Network Orchestration of end-to-end Multi-layer (OPS/OCS) Provisioning across SDN/OpenFlow and GMPLS/PCE Control Domains [5];

- Adaptive Network Manager: Coordinating Operations in Flex-grid Networks [6];

- $\quad \mathrm{iONE}$ - An environment for experimentally assessing in-operation network planning [7].

\section{CHALLENGES AND FUTURE DIRECTIONS}

A variety of industry and technology challenges remains. The protocol solutions discussed in this paper are immature and will require further investigation and development before they can be operationalised and used by operators. New forwarding mechanisms lack sufficient security and operational considerations required for complex and large scale environments. The following sub-sections outline areas of new research and their importance for SDN transport networks.

\subsection{Resource Modeling}

Suitable data models, such as YANG [8], will be needed to model the management and control of software defined network components in a technology-agnostic way. These models will need to be exchanged between control interfaces to the network devices, and abstracted and exposed to high-layer applications and Cloud users.

\subsection{Network Telemetry}

Meeting the demand for dynamic resource setup and reallocation, depends on the ability to measure network performance and assess network quality using a very small set of metrics and the ability to provide network diagnosis and root cause analysis during service disruptions. In parallel, the controller must support network resource scheduling which can adapt to real-time connection setup or resizing demands.

\subsection{Security and Trust}

Attack vectors on transport nodes (and recovery and fault diagnosis), have resulted in new security issues that are specific to SDN [9]. During development and implementation of the controller, various capabilities introduced faults and risks that expose network infrastructure to threats that did not previously exist, or were ring-fenced by single OSS platforms, and are significantly more serious, with a greater potential for harm. 
A viable Trust model will also need to be developed for infrastructure control, and it will have to implement robust authentication and varying authorisation levels should be enforced at the point of application registration to the orchestrator in order to limit the exposure to misconfiguration and malicious intent.

\section{CONCLUSIONS}

Deployment and operation of high capacity inter-data center services was a major objective for customers wanting to interconnect private and public Cloud applications. Consequently, an ABNO-based software defined transport controller platform represents an effective candidate solution to play an increasingly important role in the British Telecom and Telefonica transport network evolution process. However, as this paper has outlined, a number of operational gaps will need to be investigated and addressed.

\section{ACKNOWLEDGEMENTS}

We would like to thank British Telecom and Telefonica for their continued efforts and industrious support, and the funding provided by the European Commission for funding the ACINO project (EU grant 645127). The authors would also like to thank the other researchers at the University of Bristol and University of Lancaster, and their involvement with previous transport network research and continue to investigate transport network automation via the UK EPSRC-funded project Towards Ultimate Convergence of All Networks (TOUCAN). Finally, we would also like to thank the

\section{REFERENCES}

[1] D. King and A. Farrel, “A PCE-based Architecture for Application-based Network Operations,” IETF RFC 7491, 2015.

[2] S. Vissicchio, O. Tilmans, L. Vanbever, J. Rexford, "Central control over distributed routing," in Proc. SIGCOMM15, Aug. 17-21 2015, London, UK.

[3] Hybrid Cloud Places New Demands on the Network, Forrester Consulting, Commissioned by Juniper Networks, Apr. 2014.

[4] A. Aguado, V. López J. Marhuenda, Ó. González de Dios, and J. P. Fernández-Palacios, “ABNO: A feasible SDN approach for multi-vendor IP and optical networks," Journal of Optical Communications and Networking, Feb. 2015

[5] R. Muñoz, R. Vilalta, R. Casellas, R. Martínez, F. Francois, M. Channegowda, A. Hammad, S. Peng, R. Nejabati, D. Simeonidou, N. Yoshikane, T. Tsuritani, V. López, and Achim Autenrieth, "Experimental assessment of ABNO-based network orchestration of end-to-end multi-layer (OPS/OCS) provisioning across SDN/OpenFlow and GMPLS/PCE control domains," in Proc. European Conference on Optical Communication (ECOC), Sep. 2014.

[6] V. Lopez, O. Gerstel, R. Casellas, A. Farrel, D. King, S. López-Buedo, and J. Fernandez-Palacios, "Adaptive network manager: Coordinating operations in flex-grid networks," in Proc. Transparent Optical Networks (ICTON), Jun. 2013.

[7] L. Gifre, N. Navarro, A. Asensio, M. Ruiz, and L. Velasco, "ione: An environment for experimentally assessing in-operation network planning," in Proc. Transparent Optical Networks (ICTON), Jul. 2015.

[8] B. Claise and C. Moberg, "YANG Model Classification," Internet Draft, RFC, Apr. 2016.

[9] S. Scott-Hayward, G. O'Callaghan, and S. Sezer, "SDN security: A survey," in Proc. Future Networks and Services (SDN4FNS), Nov. 2013. 